\title{
REWARD OR ITS DENIAL DURING THE NEONATAL PERIOD AFFECTS ADULT SPATIAL MEMORY AND HIPPOCAMPAL PHOSPHORYLATED CAMP RESPONSE ELEMENT-BINDING PROTEIN LEVELS OF BOTH THE NEONATAL AND ADULT RAT
}

\section{A. DIAMANTOPOULOU, A. STAMATAKIS, T. PANAGIOTAROPOULOS ${ }^{1}$ AND F. STYLIANOPOULOU*}

Laboratory of Biology-Biochemistry, Faculty of Nursing, School of Health Sciences, University of Athens, Papadiamantopoulou 123, GR11527, Athens, Greece

\begin{abstract}
Early life experiences, particularly mother-infant interactions, have been shown to influence adult coping and learning abilities via gene-environment interactions. We have developed a paradigm, in which mother contact is used as either a positive or a negative reinforcer in a T-maze, during postnatal days 10-13. In both neonates receiving (RER) or denied (DER) the expected reward, exposure to the memory test in the absence of the mother resulted in a remarkable increase in the number of PCREB immunopositive cells, when compared to their corresponding levels $2 \mathrm{~h}$ after the completion of the training process, but also to the levels of naïve animals. In the CA3 area, the pattern of pCREB immunoreactivity, when evaluated $2 \mathrm{~h}$ after the completion of the training on postnatal day 13 seemed to distinguish between the two different neonatal experiences in the T-maze, with the DER pups showing higher levels of pCREB immunopositive cells than the RER. Exposure to the Morris Water Maze (MWM) during adulthood revealed a memory advantage of the DER animals compared to the RER and the animals not exposed to the neonatal experience. Relevantly, in the DER animals an increased number of pCREB immunopositive cells was observed in the CA3 area even $24 \mathrm{~h}$ after the end of MWM training. When also measured after exposure to the probe trial, the number of pCREB immunopositive cells was again higher in the DER compared to the RER animals. In conclusion, we show that a learning experience involving discrepancy during the particularly plastic neonatal period is able to induce long-term effects, which result in enhanced adult hippocampal dependent spatial memory. Furthermore, our data document a role of plasticity molecules like pCREB in mediating hippocampal dependent learning and detection of novelty not only in adulthood, but also more importantly in the neonatal period of the rat. @ 2011 IBRO. Published by Elsevier Ltd. All rights reserved.
\end{abstract}

\footnotetext{
${ }^{1}$ Present address: Max Planck Institute for Biological Cybernetics, Spemannstrasse 38, D 72076, Tübingen, Germany.

*Corresponding author. Tel: +30-210-7461467; fax: +30-210-7461489. E-mail address: fstilian@nurs.uoa.gr (F. Stylianopoulou).

Abbreviations: ANOVA, analysis of variance; b.w., body weight; CREB, CAMP response element-binding protein; DAB, 3, 3'-diaminobenzidine; DER, denied expected reward; LSD, least square difference; MWM, Morris water maze; NAN, naïve as neonates; NGS, normal goat seruml; NRS, normal rabbit serum; pCREB, phosphorylated cAMP response element-binding protein; PKA, protein kinase $A$; PBS, phosphate buffered saline; PND, postnatal day; RER, receiving expected reward.
}

Key words: neonatal experience, learning, novelty detection, pCREB, Morris water maze, hippocampus.

The impact of early life experiences on brain development and behaviour in adulthood has been widely investigated for decades through research in the interdisciplinary field of developmental psychobiology, pointing out its importance for shaping mental well being. Adverse experiences early in life have been linked to various psychopathologies manifested in adulthood (Breier et al., 1988; De Bellis, 2001; Heim et al., 2010; Rutter and Maughan, 1997). Furthermore, a number of studies have concluded that geneenvironment interactions mediate the determination of adult coping and cognitive behaviour (Bagot and Meaney, 2010; Godfrey et al., 2007; Kendler, 2010; Schwandt et al., 2010). Notably, the main mediator of all environmental stimulation of the pups is maternal behaviour (Leon, 1992), and the quality of the bond between the mother and the infant determines its adult behavioural phenotype (Denenberg, 1963). This has been extensively documented in rodents (Levine, 1967, 2001; Moriceau et al., 2009), primates (Berman et al., 1994; Harlow, 1965; Nelson et al., 2009; Suomi et al., 1970) and humans (Bowlby, 1954; Weinfield et al., 2000). While infants experience various challenges early in life, their brain is being developed and the neurocircuits are rewired to cope with the demands of the future environment (Lippmann et al., 2007; McEwen, 2001; Ruedi-Bettschen et al., 2006). In these terms, early life events prepare infants for a challenging environment in adulthood, or prime them for psychopathology (LyonsRuth et al., 2006; Niwa et al., 2010).

In the current literature, most of the studies examining learning and memory during early postnatal development are focusing on aversive olfactory conditioning learning (Moriceau and Sullivan, 2005, 2006; Moriceau et al., 2006; Shionoya et al., 2006; Sullivan et al., 2000). In an effort to investigate an additional aspect of the neonatal learning repertoire, we have developed a paradigm that uses the variation in reinforcement of the neonate's behaviour, in which mothercontact is used as either a positive (rewarding) or negative (frustrative) reinforcer in a T-maze, during postnatal days 10-13 (Panagiotaropoulos et al., 2009). Immediate effects of this early life experience in the behavioural and cellular level showed that pups receiving the expected reward (RER) developed a procedural-like memory, while those trained under denial of the expected reward (DER) learned to make the correct choice in the 
T-maze, albeit less efficiently than pups receiving the expected reward. Expression of the information learned by the pups denied the expected reward was contingent upon the presence of the mother in the experimental setup in exactly the same configuration as during the training. Interestingly, c-Fos immunohistochemistry revealed increased activation of the CA1 area of the hippocampus, following training under frustrative non-reward (DER).

The transcription factor CAMP response element-binding protein (CREB) has been shown to possess an essential role in long-term memory formation (Frank and Greenberg, 1994; Lamprecht, 1999; Yin and Tully, 1996). Several studies have demonstrated that CREB activation through phosphorylation and CREB-dependent gene expression occur after step-down inhibitory avoidance training (Bernabeu et al., 1997; Izquierdo et al., 2002; Taubenfeld et al., 1999), in a fear conditioning task (Hall et al., 2001; Kida et al., 2002) and novelty training (Vianna et al., 2000). In addition pCREB levels have been shown to be associated with the detection of a novel environment (Izquierdo et al., 2001; Winograd and Viola, 2004). Furthermore, activation of the PKA/CREB signalling pathway in the hippocampus plays an important role in spatial memory formation, in a radial arm maze (Mizuno et al., 2002). However, there are only a few studies on the role of pCREB in reflecting the effects of early life experiences in the brain during the neonatal period. It has been shown that $p C R E B$ levels increase in the hippocampus after an early handling experience on postnatal day 1 (Garoflos et al., 2005), while another study, documented CREB induction in the postnatal hippocampus following maternal separation (Nair et al., 2007). Given pCREB's cardinal role in memory consolidation (Athos et al., 2002; Impey et al., 1998; Montminy, 1997b; Yin et al., 1994), and novelty detection (Izquierdo et al., 2001; Winograd and Viola, 2004), we hypothesised that hippocampal pCREB levels could reflect differences in the performance of our experimental neonatal rats in the T maze. In addition, we postulated that $\mathrm{pCREB}$ would play a role in modulating the effects of the early experience in the neonatal brain. For that reason, $p C R E B$ levels were determined immunohistochemically after the completion of the learning process and after the memory test in the neonatal brain.

It has been well documented that early life events modulate adult cognitive performance in various learning tasks (Hulshof et al., 2011; Richards and Wadsworth, 2004; Schable et al., 2007; Tang, 2001; Tang et al., 2006; Vallee et al., 1999). Based on the above we hypothesised that the neonatal learning experience in the T-maze would affect learning and memory, particularly in a task involving the hippocampus, since this brain area has been shown to be affected by the neonatal experience of the T-maze (Panagiotaropoulos et al., 2009). Therefore, we studied the longterm effect of a rewarding or a frustrating neonatal learning experience on spatial learning and memory in adulthood, by employing the Morris water maze. In this paradigm the removal of the submerged platform which is usually employed in memory retrieval tests, can be viewed as a challenge bearing similarities to the neonatal frustrating experience, or even as an unexpected, frustrating event involving discrepancy in an otherwise smooth, regular sequence of reinforcing events (Schulz et al., 2004, 2007). Thus, the use of the Morris water maze permits, in addition to the evaluation of spatial learning and memory abilities, the evaluation of the effect of neonatal frustration on the response to a single frustrating event in adulthood. In addition, we determined pCREB levels in the hippocampus, after the completion of training in the Morris water maze, and after spatial memory retrieval, under the hypothesis that differences in performance in this task could be reflected in the levels of this plasticity-related transcription factor.

Taking into account all the above, the present work is based on two hypotheses: (a) Hippocampal pCREB levels of the 13-day-old pups could be related to their behaviour in the T-maze; (b) The early experience, involving spatial navigation, and higher activation of the hippocampus in the DER pups, could have long term consequences and affect the performance of the animals, as adults, in the Morris Water maze, a hippocampus dependent spatial navigation task, as well as the number of pCREB immunopositive cells in their hippocampus.

\section{EXPERIMENTAL PROCEDURES}

\section{Animals}

Wistar rats of both sexes born and reared in our laboratory were kept under standard conditions $\left(24{ }^{\circ} \mathrm{C}, 12: 12 \mathrm{~h}\right.$ light/dark cycle) and received food and water ad libitum. Prior to the day of birth, which was designated as postnatal day 0 , each litter was assigned to either of the two experimental groups (pups denied or receiving the expected reward), or to the naïve (non-handled) group. In order to maintain stable environmental stimulation for the pups, instead of cleaning the cage, wood chip was added every 4-5 days, without distracting either the pups or the dam. On postnatal day 22 all animals to be used in the adult experiments were weaned and housed in same group, same sex cages of three to four animals per cage. All animal experiments were carried out in agreement with ethical recommendation of the European Communities Council Directive of 24 November 1986 (86/609/EEC).

\section{Neonatal training in the T-maze}

We used a custom-made T-maze whose arms led to two cages with dimensions $30 \mathrm{~cm}$ width $\times 22 \mathrm{~cm}$ length $\times 30 \mathrm{~cm}$ height. The dimensions of the start box and the arms of the T-maze were $8 \mathrm{~cm}$ width $\times 6 \mathrm{~cm}$ length $\times 6 \mathrm{~cm}$ height and $7 \mathrm{~cm}$ width $\times 30 \mathrm{~cm}$ length $\times 6$ $\mathrm{cm}$ height, respectively. At the end of the right arm of the T-maze a small sliding door $\left(9 \times 11 \mathrm{~cm}^{2}\right)$ permitted access to the mothercontaining cage when pups were trained under continuous reinforcement or remained always closed, preventing entrance in the cage, when pups were trained under continuous frustration. At the end of the left arm of the T-maze, another cage was placed, without access from inside the T-maze, containing a virgin female rat for control purposes.

Animals were trained in this T-maze during postnatal days 1013. This particular age span was selected on one hand because before postnatal day 10 animals have very little motor activity, and on the other hand, after day 13 eye opening occurs, and we wanted to perform the training in the absence of visual cues.

Continuous reinforcement training (receiving expected reward-RER rats). Rats trained under the continuous reinforcement schedule were exposed to 10 trials per day (which amounts to a total of 40 trials for the four experimental days), as previously 
described (Panagiotaropoulos et al., 2009). At the end of the duration of the trial, or until the pup located the entrance of the mother-containing cage the sliding door opened and the mother could retrieve the pup. If a pup did not succeed to reach the entrance of the mother-containing cage before the end of the $60 \mathrm{~s}$ (maximum duration of the trial), it was gently guided to the entrance, the sliding door opened and the mother was allowed to retrieve the pup. In both cases, following the end of the trial, the pup returned to the mother-containing cage and then the next pup was exposed to the same procedure. When all pups were exposed to the first trial, the same procedure was repeated for the next trial until the end of the experimental session consisting of 10 trials.

Continuous non-reinforcement training (denied expected reward-DER rats). The same procedure was followed for DER rats (10 trials per day for 4 training days, $60 \mathrm{~s}$ each). The behavioural observation was terminated at the time the pup located the entrance of the mother-containing cage. If a pup did not succeed to reach the entrance of the mother-containing cage before the end of the $60 \mathrm{~s}$ (maximum duration of the trial), it was gently guided to the entrance and had to remain there for $20 \mathrm{~s}$. Immediately after the end of the trial, the rat was returned to the start box of the T-maze for the next trial. Following the completion of 10 trials, the pup was returned to the mother-containing cage and then another pup was exposed to the same procedure. At the end of the 10 daily trials for all the pups, the mother, followed by the litter, was returned to the home cage in the animal facility.

\section{Spatial memory retrieval in the absence of maternal cues}

At postnatal day $13,2 \mathrm{~h}$ after their exposure to the last training trial, pups were exposed to a single retrieval trial in the absence of mother-elicited stimuli. Both cages (containing the mother of the pups and the control female animal) were removed from the experimental set up and the pups were placed for $60 \mathrm{~s}$ in the T-maze.

Spatial memory retrieval in the presence of maternal cues. Another cohort of DER rats was tested at the same time point (2 $\mathrm{h}$ after the last training trial on postnatal day 13) for retrieval in the presence of mother-elicited stimuli in two different positions. The mother was located either in the same position as during training (in the right arm of the T-maze) or in the exact opposite arm (left) of the T-maze.

Spatial learning and memory retrieval during adulthood. Neonatally DER and RER as well as naïve as neonates (NAN) rats were tested for spatial learning and memory retrieval in adulthood (3-4 months old) in the Morris water maze (MWM) (29 DER, 30 RER and 29 NAN animals). The MWM consisted of a circular tank (1.38 m in diameter, $0.5 \mathrm{~m}$ in height) filled to a depth of $28 \mathrm{~cm}$ with tap water $(\mathrm{T}=24 \pm 1 \mathrm{C}$ ) made opaque with milk. During the 4 days of training, a submerged platform $\left(13 \times 13 \mathrm{~cm}^{2}\right)$ was placed in a fixed position, 2 $\mathrm{cm}$ beneath the water surface. Each day of training consisted of four consecutive trials, each from a different starting point relative to the platform (which was in place), with an inter-trial interval of $30 \mathrm{~s}$. When a rat located the platform, it was allowed to remain on it for $30 \mathrm{~s}$. If the rat could not locate the platform within $90 \mathrm{~s}$ (the maximum duration of a trial), it was gently guided to it and allowed to remain there for $30 \mathrm{~s}$. The classic long-term memory retrieval trial (which in our study also represents a frustrating event) was performed $24 \mathrm{~h}$ after the last training session and consisted of a $60 \mathrm{~s}$ free swim trial in the absence of the platform. Rats were released in the water maze from a position exactly opposite from the target quadrant. In order to assess spatial learning we calculated the mean distance moved as well as escape latency to the platform for each day of training. Long-term memory retrieval was assessed by the percent of distance moved and time spent in the target quadrant (the one in which the platform was located during training) relative to that in the other three quadrants.
For both spatial learning and retrieval, data from both male and female animals were grouped since we did not observe any statistically significant sex difference (data not shown).

\section{Analysis of behaviour}

Behaviour was digitally recorded at a frequency of $2-5 \mathrm{~Hz}$ for the $\mathrm{T}$-maze experiments and at $2 \mathrm{~Hz}$ for the water maze experiment using the Noldus Ethovision system (Ethovision 3.0, Noldus Information Technologies, Wageningen, the Netherlands).

\section{Determination of hippocampal pCREB1 and total CREB1 levels}

Immunohistochemical analysis was performed on randomly selected PND 13 brains from the same cohorts of animals whose behavioural data have been reported previously (Panagiotaropoulos et al., 2009).

Animals were sacrificed $2 \mathrm{~h}$ after the last training trial on postnatal day 13 (at the time point of the retrieval trial) (nine RER, nine DER and seven naïve animals) or immediately after the memory retrieval trial, $2 \mathrm{~h}$ after the last training trial, on postnatal day 13 ffive RER, 15 DER [five had undergone the probe trial in the absence of the mother (standard No mama, NM probe trial), five the probe trial with the mother in the inversed position and five the probe trial with the mother in the usual position as during training] and seven naïve animals\}. Data from both genders were pooled since no significant gender differences were observed.

Hippocampal pCREB1 and total CREB1 levels were also determined immunohistochemically in the brain of adult animals neonatally maintained as naïve (NAN) as well as subjected to either continuous reward (RER) or continuous non-reward (DER). Animals were sacrificed (a) $24 \mathrm{~h}$ after the end of the 4-day training (five RER, five DER and five NAN animals) or (b) immediately following the retrieval trial of the Morris water maze, which was performed $24 \mathrm{~h}$ after the end of the 4-day training (five RER, five DER and five NAN animals). Immunohistochemical determination of pCREB1 and total CREB1 levels in the adult hippocampus was performed on brains from randomly selected animals from the cohort subjected to the behavioural analysis in the MWM.

Tissue preparation. Pups were anesthetised with chloral hydrate (injected i.p. at a dose of $400 \mathrm{mg} / \mathrm{kg} \mathrm{b.w.)} \mathrm{and} \mathrm{transcar-}$ dially perfused with $20-30 \mathrm{ml}$ of ice-cold $4 \%$ paraformaldehyde (or $20 \mathrm{ml}$ of saline followed by $80-100 \mathrm{ml}$ of paraformaldehyde for adult rats) in phosphate buffer $(0.1 \mathrm{M}, \mathrm{pH}=7.4)$. Brains were isolated, postfixed overnight in the perfusion medium at $4{ }^{\circ} \mathrm{C}$, further processed and finally embedded in paraffin. Serial sagittal sections $(6 \mu \mathrm{m})$ were cut and mounted on silane-coated slides.

Immunohistochemistry and quantification of immunopositive cells. Sections were deparaffinised, rehydrated, and subsequently subjected to antigen retrieval in a microwave oven for $15 \mathrm{~min}$ in citrate buffer $(10 \mathrm{mM}, \mathrm{pH}=6.0)$. Following washing with phosphatebuffered saline (PBS) containing $0.4 \%$ Triton X-100 (SERVA, Heidelberg, Germany), slides were incubated in PBS containing $0.4 \%$ Triton X-100 and either $10 \%$ normal rabbit serum (NRS) or $10 \%$ normal goat serum (NGS) (DakoCytomation, Glostrup, Denmark) for $1 \mathrm{~h}$ at room temperature. Afterwards, sections were incubated overnight at $4{ }^{\circ} \mathrm{C}$ with a goat polyclonal anti-pCREB1 antibody (Santa Cruz, Santa Cruz, CA, USA) or a rabbit polyclonal anti-CREB1 antibody (Santa Cruz, Santa Cruz, CA, USA) at a concentration of 1 $\mu \mathrm{g} / \mathrm{ml}$ diluted in PBS containing $0.4 \%$ Triton X-100 and either $4 \%$ NRS or $4 \%$ NGS. Following incubation with the primary antibody, slides were washed in PBS and incubated for $2 \mathrm{~h}$ at room temperature with either a biotinylated rabbit anti-goat (DakoCytomation, Glostrup, Denmark) or a biotinylated goat anti-rabbit antibody (DakoCytomation, Denmark) at a concentration of $5 \mu \mathrm{g} / \mathrm{ml}$ diluted in PBS containing $2 \%$ NGS. Following several rinses in PBS, slides were exposed to the $A B C$ reagent (DakoCytomation, Glostrup, Denmark) 
for 60 min at room temperature. Slides were then washed in PBS and stained with 3,3'-Diaminobenzidine (DAB) $(1.7 \mathrm{mM}$, Sigma-Aldrich, St. Louis, MO, USA) diluted in Tris-HCl buffer $(10 \mathrm{mM}, \mathrm{pH}=7.6)$ and $0.03 \% \mathrm{H}_{2} \mathrm{O}_{2}$ for $2-5 \mathrm{~min}$ at room temperature. Finally, they were washed, dehydrated, and coverslipped with DePex (SERVA, Heidelberg, Germany) and analysed microscopically under a brightfield microscope.

The image analysis program "Image Pro Plus" (Media Cybernetics, Bethesda, MD, USA) was used for counting cells immunopositive for pCREB1 in the CA1 and CA3 hippocampal areas. Cell counting was performed "blindly" by two independent investigators. A "threshold" was set in the image analysis system, in order to include in the counting only cells stained above a certain, preset degree. This "threshold" was defined at a grey value equal to 90 , based to the background staining observed in the sections processed in the absence of primary antibody. The mediolateral extent of the hippocampus in which cell counting was performed, was first defined by employing Cresyl-Violet stained sections. For the PND13 brain it corresponds to Mediolateral stereotaxic coordinates $=0.6-0.85 \mathrm{~mm}$, according to the anatomical atlas of Ramachandra and Subramanian (2011), and for the adult brain to stereotaxic coordinates Mediolateral $=1.4-1.8 \mathrm{~mm}$, according to the anatomical atlas of Paxinos and Watson (1986). For each antigen ( $p C R E B$ and CREB) positive cells were counted in a mediolateral series of equally spaced sections [four to six sections, spaced by $30 \mu \mathrm{m}$ (one section counted for every five $6 \mu \mathrm{m}$-sections cut)], starting from a randomly selected section containing the brain area of interest. In each brain section, cells were counted in two different optical fields (rectangular of fixed size) of each hippocampal area investigated. For each animal, the number of positive cells per optical field was the average value calculated from the data from all optical fields in all brain sections evaluated.

\section{Statistical analysis}

Behavioural data were analysed using one or two-way analysis of variance (ANOVA) with or without repeated measures, as appropriate. Least square difference (LSD) posthoc statistical tests where used when necessary. Two-way ANOVA was also used for the analysis of the immunohistochemical data.

\section{RESULTS}

\section{In the neonatal hippocampus, differences in reinforcement lead to region specific alterations in the number of pCREB immunopositive cells}

It is widely known that pCREB is a molecule important for plasticity, novelty detection and learning processes. Previous results (Panagiotaropoulos et al., 2009) showed an involvement of the hippocampus during neonatal training in a T-maze. This led us to hypothesise, that the levels of pCREB in the hippocampus of neonates exposed to differences in reinforcement in the T-maze would give further insight into the underlying cellular information processing mechanisms employed by the two experimental groups. Evaluation of pCREB expression in the CA1 and CA3 areas of the hippocampus showed a distinct pattern of activation, depending on the experience during training in the T-maze and the exposure or not to the memory test without the presence of the mother. In particular, in the CA1 area of neonatal rat at PND13, there was a main effect of the condition (i.e. memory test-no memory test) $\left(F_{1,28}=96.364\right.$, $P<0.001)$ and an interaction between the condition and the animal group $\left(F_{2,28}=25.374, P<0.001\right)$. More specifically, in both the RER $\left(F_{1,10}=209.443, \quad P<0.001\right)$ and DER
$\left(F_{1,10}=10.362, P=0.011\right)$ pups exposure to the memory test in the absence of the mother resulted in a remarkable increase of pCREB immunopositive cells, compared to their levels $2 \mathrm{~h}$ after the completion of the training process, as well as the levels of naive pups $\left(F_{2,13}=26.514, P<0.001\right.$, Fig. $1 A)$. There were no statistically significant differences in the number of pCREB immunopositive cells in the CA1 area, between the RER and DER pups, either without exposure to the probe, or after the probe.

As reported previously (Panagiotaropoulos et al., 2009), the performance of the DER pups in the memory test was contingent upon the presence of the mother in the same configuration as during training in the T-maze, which we hypothesised could be reflected in the levels of $p C R E B$ expression. Interestingly, exposure of the DER pups to this type of memory test, with the mother being present at the exact same position as during training, resulted in significantly lower number of pCREB immunopositive cells in the CA1 hippocampal area $\left(F_{2,12}=4.176, P=0.048\right)$, compared to those after the other two types of memory test employed (absence of the mother, or with the mother at the opposite arm as the one during training) (Fig. 1B).

In contrast to the CA1 hippocampal area, in the CA3 area, the pattern of pCREB immunoreactivity, when evaluated $2 \mathrm{~h}$ after the completion of the training on PND13 seemed to distinguish between the two different neonatal experiences in the T-maze (Fig. 1B). Two-way ANOVA revealed a significant condition (i.e. memory test-no memory test $) \times$ group interaction in the CA3 $\left(F_{2,32}=6.820\right.$, $P=0.004)$. More specifically, $2 \mathrm{~h}$ after training the RER pups showed lower levels of pCREB immunopositive cells in the CA3 $\left(F_{2,17}=3.934, P=0.042\right)$ hippocampal area, than both the DER and the naïve pups. When subjected to the probe in the absence of the mother pups exhibited in the CA3 hippocampal area an increase [RER $\left(F_{1,11}=34.929\right.$, $P<0.001)$ and DER $\left.\left(F_{1,10}=6.571, P=0.031\right)\right]$ in the number of pCREB immunopositive cells, compared to the condition $2 \mathrm{~h}$ after the completion of training on PND13 (without exposure to the probe test), reaching statistically significant higher levels than those of naive pups $\left(F_{2,14}=5.910, P=0.016\right)$.

It should be noted that the number of total CREBpositive cells in the hippocampus did not differ between PND13 naive animals and those exposed to neonatal training (CA1: DER 102 \pm 5 , RER $104 \pm 4$ and naive $109 \pm 6$; CA3: DER $59 \pm 5$, RER $58 \pm 7$ and naive $56 \pm 2$ ).

\section{Differences in reinforcement during the neonatal period have long lasting effects on a cognitive task in adulthood: animals denied the expected reward exhibit enhanced memory in the Morris water maze task}

Since early life events have often long-term consequences on brain development and adult cognitive abilities, we argued that the experience in the T-maze during postnatal days 10-13, would affect adult performance in a cognitive task, particularly one involving the hippocampus. We thus exposed adult neonatally DER, RER or naïve animals to the MWM, a hippocampus-dependent task. In the learning phase of this task, there was a significant reduction in 
I.

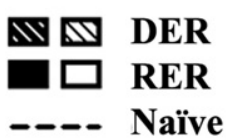

A.

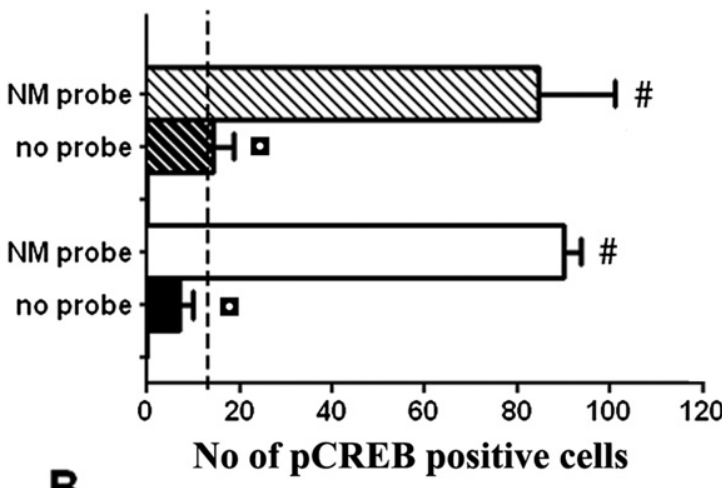

B.

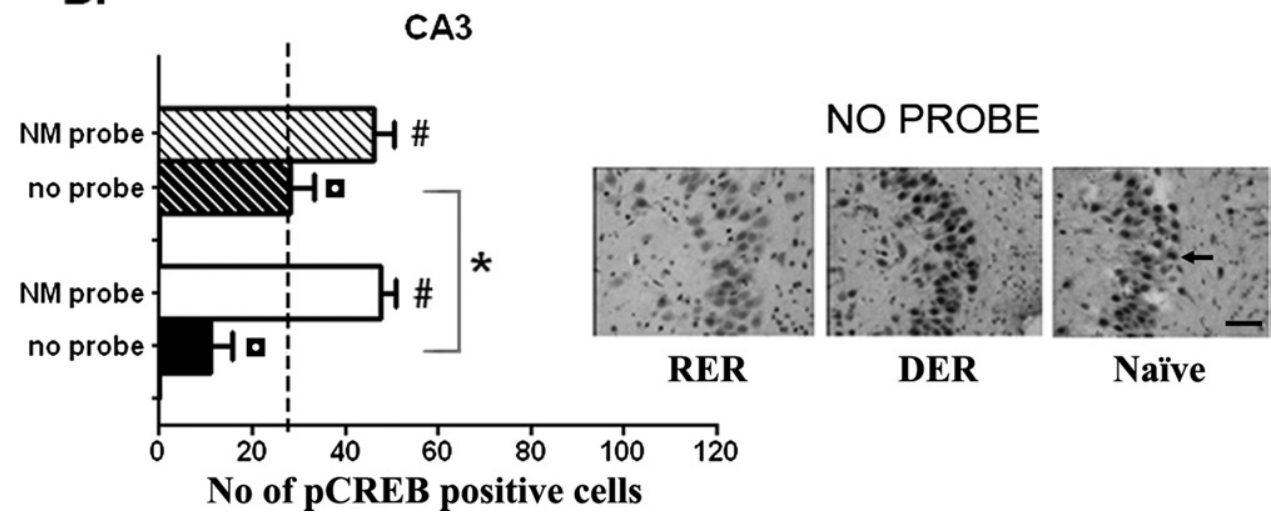

II.
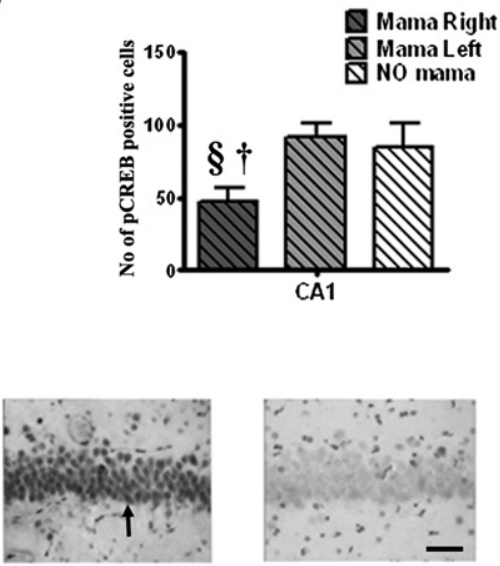

PROBE

Fig. 1. (I) Quantification of pCREB immunopositive cells, $2 \mathrm{~h}$ after the last training trial on postnatal day 13 and immediately after the NM probe trial (i.e. in the absence of the mother), on the same day, in the CA1 and CA3 hippocampal areas of DER, RER and naïve rat pups. pCREB immunoreactivity measured immediately after the probe trial on postnatal day 13 was higher both in DER and RER pups, when compared to pCREB immunoreactivity measured $2 \mathrm{~h}$ after the last training trial, on the same day, in the CA1 (A DER: $P<0.0001, \mathrm{RER}: \mathbf{0} P<0.0001$ ) and CA3 (B DER: - $P<0.031$, RER: $P<0.0001)$ area of the hippocampus. The number of pCREB immunopositive cells of DER $(n=5)$ and RER $(n=5)$ pups was higher than that of naïve $(n=7)$ rat pups (dashed line) when measured immediately after the NM probe trial in the CA1 (A DER vs. naïve: posthoc, ${ }^{\#} P<0.001$; RER vs. naïve l: posthoc, ${ }^{\#} P<0.001$ ), and the CA3 (B DER vs. naïve: posthoc, ${ }^{\#} P=0.015$; RER vs. naïve: posthoc, ${ }^{\#} P=0.009$ ) hippocampal area. In addition, pCREB immunoreactivity was lower in RER $(n=9)$ compared to DER $(n=9)$ (posthoc, $\left.{ }^{*} P=0.027\right)$ and naïve $(n=7)$ (posthoc, $\left.P=0.036\right)$ pups in the CA3 (B), when evaluated $2 \mathrm{~h}$ after the last training trial on postnatal day 13. (II) The number of pCREB immunopositive cells in the CA1 area was lower in the DER rat pups that had undergone the probe trial with the mother at the same location as during the training $(n=5)$, compared to that measured when pups had undergone the probe trial in the absence of the mother $(n=5)$ (posthoc, $\$ P=0.05$ ) or the one where the mother was in the opposite arm of the T-maze as during training $(n=5)$ (posthoc, $\left.{ }^{\dagger} P=0.025\right)$. Bar graphs represent means \pm SEM. Representative photomicrographs showing pCREB immunoreactivity immediately after the probe trial on postnatal day 13 versus $2 \mathrm{~h}$ after the last training trial on postnatal day 13 in the CA1 hippocampal area of the RER pups (A), and in the hippocampal CA3 area of RER, DER and naïve rat pups $2 \mathrm{~h}$ after the last training trial on postnatal day 13 (B). Arrows indicate representative pCREB immunopositive cells included in the quantification of the results (scale bar: $50 \mu \mathrm{m}$ ).

escape latencies $\left(F_{3,246}=100.442, P<0.0001\right)$ and total distance moved $\left(F_{3,246}=89.636, P<0.0001\right)$ across the four consecutive days of training, for all groups (RER, DER, NAN), but the learning curves did not differ among them (Fig. 2A). However, the memory test in the absence of the hidden platform, conducted $24 \mathrm{~h}$ after the last training trial, revealed a memory advantage of the DER animals compared to the RER and NAN. Both the time they spent in the target quadrant $\left(F_{2,52}=3.226, P=0.048\right)$, as well as the percent of the total distance moved in the target quad- rant $\left(F_{2,52}=3.294, P=0.045\right)$ were higher in the DER animals (Fig. 2B).

The enhanced memory of animals denied the reward as neonates could be related to their sustained pCREB expression in the CA3 area, even $24 \mathrm{~h}$ after the end of training in the Morris Water Maze, in adulthood

Various studies have shown than PCREB levels increase after the end of the learning process in multiple learning 
A.

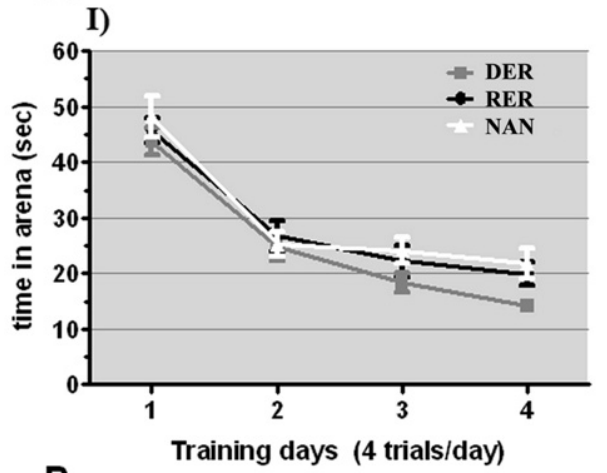

B.

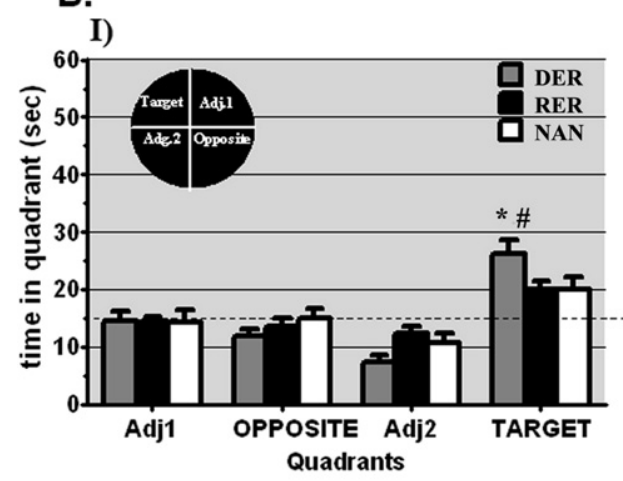

II)

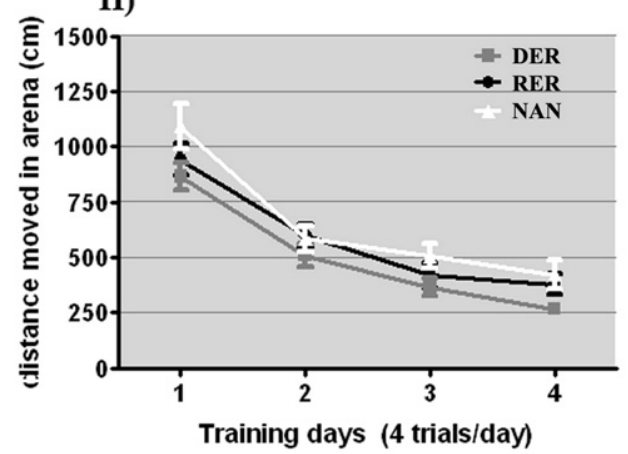

II)

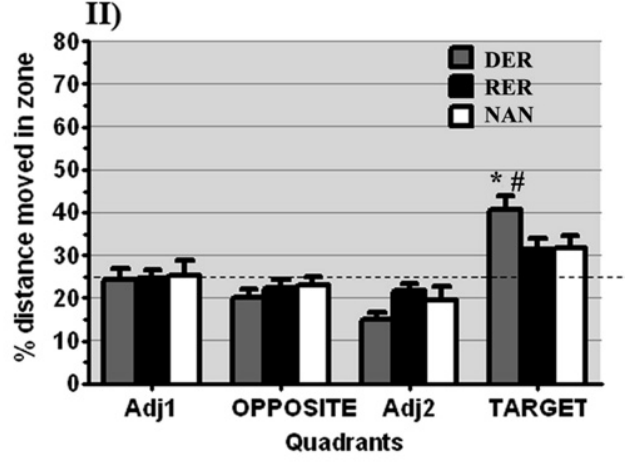

Fig. 2. (A) (I) Escape latencies and (II) total distance covered to find the hidden platform during spatial learning (blocks of four trials/d) in the MWM. (B) (I) Time spent in each quadrant and (II) percent of distance moved within each quadrant during the spatial memory probe, $24 \mathrm{~h}$ after the end of the MWM training. Bars represent means \pm SEM. ${ }^{*} P<0.05$ (statistically significant difference between DER $(n=29)$ and RER $(n=30)$ animals), \# $P<0.05$ (statistically significant difference between DER $(n=29)$ and naïve as neonates (NAN, $n=29)$ animals). Dotted lines represent performance at chance levels.

tasks. Increase of hippocampal pCREB levels has been proposed to be linked to the processing and consolidation of contextual and spatial information (Impey et al., 1998; Mizuno et al., 2002). Our results showed that in DER animals, there was a significant maintenance of increased pCREB expression-compared to both the RER and the NAN animals-even $24 \mathrm{~h}$ after the end of MWM training, in both the CA1 and CA3 hippocampal areas. More specifically, in the CA1 area the numbers of pCREB immunopositive cells were higher in the DER compared to the RER and NAN animals $\left(F_{2,12}=20.060, P<0.001\right.$; posthoc $P=$ 0.004 and $P<0.001$, respectively) as well as in the RER compared to the NAN (posthoc $P=0.042$ ). Moreover, in the CA3 $\left(F_{2,12}=10.845, P=0.003\right)$, pCREB activation was significantly higher only in the DER animals, according to the posthoc analysis (Fig. 3). This finding could be related to the superior mnemonic abilities of the DER animals, since the CA3 hippocampal area has been shown to be specifically involved in spatial pattern separation, association and completion (Kesner et al., 2004; Gold and Kesner, 2005).

Interestingly, when also measured after exposure to the probe trial, the numbers of pCREB immunopositive cells were even higher in the DER compared to the RER animals: $300 \%$ in the CA1 $\left(F_{1,12}=5.456, P=0.039\right)$ and $400 \%$ in the CA3 $\left(F_{1,12}=6.438, P=0.026\right)$. The fact that DER animals exhibited sustained elevated pCREB levels in the CA1 and CA3 areas of the hippocampus following the probe trial could be also related to their enhanced spatial memory performance.

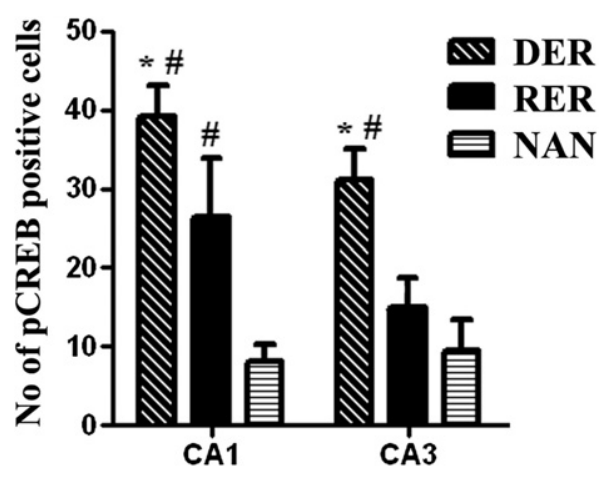

Fig. 3. Quantification of pCREB immunopositive cells, $24 \mathrm{~h}$ after the end of training in the Morris Water Maze in the CA1 and CA3 hippocampal areas of adult animals trained either as DER or as RER as neonates, as well as naïve as neonates (NAN) adult animals. In the CA1 hippocampal area, pCREB immunoreactivity was higher in DER $(n=5)$ compared to the RER $(n=5)$ (posthoc, $\left.{ }^{*} P=0.004\right)$ and NAN animals $(n=5)$ (posthoc, ${ }^{\#} P<0.001$ ) as well as in RER compared to NAN (posthoc, ${ }^{\#} P=0.042$ ). In the CA3 area, the number of pCREB immunopositive cells was higher in the DER animals compared to both the RER (posthoc, * $P=0.020$ ), and the NAN (posthoc, ${ }^{\#} P=0.001$ ) animals. 
Despite its significant effects on pCREB levels, the neonatal experience did not affect the levels of total CREB in the adult hippocampus following exposure to the MWM. There were no statistically significant differences between DER, RER or NAN animals (CA1: DER $68 \pm 6$, RER $69 \pm 2$ and NAN 71 \pm 3 ; CA3: DER $44 \pm 5$, RER $42 \pm 4$ and NAN $40 \pm 5)$.

\section{DISCUSSION}

In the present study, it was shown that differences in reinforcement during learning of a T-maze in the neonatal period of the rat are accompanied by a differential pattern of pCREB activation within the neonatal hippocampus. In addition, the early experience has long-term consequences, affecting hippocampal pCREB levels following learning of the Morris Water Maze task, and resulting in better mnemonic abilities in the animals trained as neonates under the condition of denial of the expected reward of maternal contact.

As previously described, when trained under the reward of maternal contact for 4 days (during PND10-13, i.e. before eye opening) rat pups learn by a stimulus/response strategy to associate the right arm of the T-maze with the presence of the mother, and through the repeated training develop a procedural type of memory. Even when trained under frustrative non-reward pups manage to learn the position of the mother in the T-maze, albeit less efficiently (Panagiotaropoulos et al., 2009). In the present study, we show that $2 \mathrm{~h}$ after the end of training, pCREB levels in the CA3 hippocampal area of 13-day-old pups trained under reward were lower compared to those of pups trained under the frustrating experience of inhibiting contact with the mother, whose levels did not differ from those of the naive pups. Relevant to our results could be the finding of Colombo et al. (2003) who showed in adult rats that when a stimulus-response learning strategy is employed, there is only an immediate CREB phosphorylation. If the same holds for the $\mathrm{P} 13$ rats, such a very early following training increase of $\mathrm{pCREB}$ in the neonatally rewarded pups would have been missed in our experiments.

Previous findings from our lab have shown a higher activation state of the hippocampus-as assessed by the number of c-Fos immunopositive cells in the CA1 area-of the neonatally frustrated animals compared to the rewarded (Panagiotaropoulos et al., 2009). That result represented the increased neuronal firing of approach-consummate mismatch units (O'Keefe and Nadel, 1978) activated by the experienced frustration/absence of reward when contact with the mother was denied during training. On the other hand, in the present work, which examines changes in PCREB, the difference between pups trained under reward or frustration is localised in the CA3 area. It should be noted that CREB phosphorylation occurs because of the processes going on both during and after learning, as well as of consolidation, and reflects the transcriptional activation state of the neurons at the time of the animal's sacrifice. Notably regarding the difference in localisation of the expression of c-Fos and pCREB, Colombo et al. (2003), have reported similar results showing that $1 \mathrm{~h}$ after training of adult rats in a cross maze c-Fos was increased only in the CA1 area and not the CA3, while pCREB was increased in both the CA1 and CA3.

It should be noted that although at the level of naïve pups, the number of pCREB immunopositive cells in the DER pups were higher compared to the RER. Interestingly this difference is limited to the CA3 hippocampal area, which is known to play a role in various aspects of spatial information processing, that is, pattern separation, association and completion (Kesner et al., 2004; Gold and Kesner, 2005). Studies on the role of pCREB in learning processes during the neonatal period have documented its involvement mainly in olfactory learning. More specifically it has been shown that PCREB is increased in the neonate rat olfactory bulb by maternal odour preference or odour preference-conditioned learning (McLean et al., 1999; Raineki et al., 2009). Notably our study is the first to show that $\mathrm{pCREB}$ is also involved in a different type of learning in the neonate, supporting further the global role of $\mathrm{pCREB}$ in memory formation.

Phosphorylation of CREB at Ser133 is necessary for CREB dependent transcriptional activation of genes that subsequently facilitates mnemonic processes (Montminy, 1997a). pCREB molecules are crucial for memory consolidation of newly acquired information (Abel and Kandel, 1998; Blendy et al., 1996; Frank and Greenberg, 1994; Schulman, 1995; Stevens, 1994; Wang et al., 2006; Yin and Tully, 1996) as well as the detection of novelty (Izquierdo et al., 2001), but not for memory of spatial habituation (Moncada and Viola, 2006; Winograd and Viola, 2004). Based on the above, pCREB is not expected to be involved in the behaviour of RER pups at that final stage of the neonatal learning, where all information concerning the Tmaze experience has been consolidated. Indeed the number of pCREB immunopositive cells $2 \mathrm{~h}$ after the end of training (no probe) was very low in the RER pups. On the contrary, in the DER animals, maintaining a certain level of CREB activation in the hippocampus seems to be crucial, since memory traces have not yet been stabilised and is still dependent on the stimulus driving learning (i.e. the presence of the mother in the right arm of the maze).

The different levels of pCREB immunoreactivity in the CA3 area of the hippocampus between the DER and the RER animals, at the time when the memory probe would have taken place ( $2 \mathrm{~h}$ after the end of training) could also be related to their differences in behaviour in the memory trial in the absence of the mother. According to our previously published data (Panagiotaropoulos et al., 2009), pups receiving the expected reward during training in the T-maze develop a procedural-like memory and express in the memory trial the learned information independently from the reinforcing stimulus, that is the presence of the mother. In contrast, the pups denied the expected reward show inhibition of approach behaviour in the memory trial in the absence of the mother, and expressed the learned information only in the presence of the mother in the experimental setup in exactly the same configuration as during the training. It thus appears that learning under denial 
of expected reward results in a behavioural state more sensitive to contextual changes, which require an activated hippocampus, with adequate pCREB levels available.

Based on their experience acquired during training, pups have formed an expectation of the location of the mother. When the mother is absent this could represent a surprise or novelty for the pups, and it is known that situations of novelty or unfamiliarity lead to an increase in pCREB levels (Moncada and Viola, 2006; Viola et al., 2000). Thus, the increased levels of pCREB after the memory trial in the T-maze observed in both DER and RER pups could be due to the sense of novelty and unfamiliarity they experience during the memory trial, due to the absence of the mother.

The view that conflict between expected and perceived stimuli leads to increased levels of pCREB is also supported by our data regarding $p C R E B$ levels of the DER pups following memory retrieval in the different types of probe trial. When the mother was present in the setup in the same place as during training, pCREB levels in the CA1 hippocampal area were lower than when the mother was either absent, or in the opposite arm of the maze. It can be argued that the presence of the mother in the expected location can act to relieve the sense of novelty or unfamiliarity elicited in the other two cases.

pCREB is well known to be necessary for activation of gene transcription (Liu and Graybiel, 1996). Thus, the different pCREB levels of the RER and DER pups could alter the pattern of hippocampal connections and have long-term consequences on behaviour that span through adulthood. Specifically, during the second postnatal week hippocampal circuitry is within a critical period, which defines anatomical and functional maturation. During this period the majority of afferent synaptic connections are formed in the hippocampus (Crain et al., 1973; Loy and Moore, 1979) and the granule cells of the dentate gyrus establish synaptic contacts on CA3 pyramidal cell dendrites, indicating maturation of their efferent connections. Thus, different levels of phosphorylation of CREB within this critical period could consequently modify the maturation of hippocampal circuitry through prolonged gene expression. Modification of hippocampal function can have long-term effects leading to a different behavioural phenotype during adulthood (Akers et al., 2006), as our results indeed show.

A number of studies have shown that early life adverse experiences, like maternal separation have been linked to cognitive impairments in water maze tasks (Lehmann et al., 1999; Pryce et al., 2003). On the other hand early life stimulation described as neonatal handling has been shown to restore spatial learning in aged rodents (Escorihuela et al., 1995; Meaney et al., 1988; Zaharia et al., 1996) but also enhances adult spatial learning abilities (Bilbo et al., 2007; Garoflos et al., 2005; Stamatakis et al., 2008). In our early life experience paradigm, the frustrative non-reward experience early in life results in a type of memory that appears more sensitive to contextual changes during the retrieval trial. In a recently revived view of the function of memory, plastic changes known to ac- company memory formation should not only be viewed as creating a memory trace of past experience but also as creating a capacity to prepare for future challenges (Dudai and Carruthers, 2005). Our results corroborate this hypothesis by demonstrating that the type of training the animal had experienced during the neonatal period modulates the behavioural response to a single frustrating event in adulthood (the removal of the platform in the Morris water maze). Specifically, we found that neonatal training under frustrative non-reward renders rats more persistent in exploration of the platform quadrant during the retrieval trial in the Morris water maze. It should be noted that the early experience of denial contact with the mother in our paradigm was of a short duration, and-similarly to the neonatal handling paradigm-does not interfere with the mother-pup attachment bond, and could thus be considered more as a "challenging" stimulus rather than a detrimental one.

The temporal pattern of pCREB activation following MWM training has not been extensively studied, with the exception of the detailed work of Porte et al. (2008) in normal mice. They have reported that extended (for 9 days) spatial learning induces in the CA1 hippocampal area a biphasic activation of CREB with a first peak at 15-60 min and a second at $9 \mathrm{~h}$ posttraining, while in the CA3 area the second peak was not observed. Furthermore, these authors have shown that there is persistent phosphorylation of CREB in both the CA1 and CA3 areas of the hippocampus, following the extensive training.

Our results show that $24 \mathrm{~h}$ following a 4-day training protocol, the adult DER animals, which exhibit better mnemonic abilities, have higher numbers of pCREB immunopositive cells in the CA3 area, than both the RER and the NAN, while there is no statistical difference between RER and NAN animals. It is particularly interesting that it is in the CA3 area, which supports spatial pattern separation, association and completion (Kesner et al., 2004; Gold and Kesner, 2005), that these differences in pCREB are observed. Sustained increase in CREB phosphorylation $24 \mathrm{~h}$ following training and right after the single frustrating event experienced during retrieval in the MWM in adulthood in the DER animals could accompany the ongoing information processing, as an index of enhanced behavioural plasticity of these animals. In support of the need for increased phosphorylation of CREB when changes in the environment occur, it has been shown that such an increase in the hippocampus has been linked to detection of spatial novelty (Moncada and Viola, 2006; Winograd and Viola, 2004), but not familiarity.

\section{CONCLUSION}

In conclusion, the present work is the first to document a role of plasticity molecules like pCREB in mediating hippocampal dependent learning and detection of novelty not only in adulthood, but also more importantly in the neonatal period of the rat. Furthermore, our results provide experimental evidence that a learning experience involving discrepancy during a particular plastic period like the neonatal 
age is able to induce long-term effects, which result in enhanced hippocampal dependent spatial memory as well as increased hippocampal activation by a single event involving discrepancy in adulthood. Interestingly, in a study in humans, children who were delayed gratification longer in laboratory situations, were found to cope better with frustration and stress in adulthood (Mischel et al., 1989). In other words, it can be argued that regarding the DER animals our data are in line with the match/mismatch theory or "predictive adaptive response", which supports that, adverse early life events could prepare individuals for future events of adversity in adulthood (Champagne et al., 2009; Godfrey et al., 2007; Oomen et al., 2010).

Acknowledgments-This work was supported by the National Strategic Reference Framework (NSRF), Project Iraklitos II, cofunded by the European Funds for Cohesion Policy and National Funds and by research grants from the Special Account for Research Grants of the University of Athens. We thank T. Moressis for assisting in the immunohistochemistry studies.

\section{REFERENCES}

Abel T, Kandel E (1998) Positive and negative regulatory mechanisms that mediate long-term memory storage. Brain Res Brain Res Rev 26:360-378.

Akers KG, Nakazawa M, Romeo RD, Connor JA, McEwen BS, Tang AC (2006) Early life modulators and predictors of adult synaptic plasticity. Eur J Neurosci 24:547-554.

Athos J, Impey S, Pineda VV, Chen X, Storm DR (2002) Hippocampal $\mathrm{CRE}$-mediated gene expression is required for contextual memory formation. Nat Neurosci 5:1119-1120.

Bagot RC, Meaney MJ (2010) Epigenetics and the biological basis of gene $x$ environment interactions. J Am Acad Child Adolesc Psychiatry 49:752-771.

Berman CM, Rasmussen KL, Suomi SJ (1994) Responses of freeranging rhesus monkeys to a natural form of social separation. I. Parallels with mother-infant separation in captivity. Child Dev 65 : 1028-1041.

Bernabeu R, Bevilaqua L, Ardenghi P, Bromberg E, Schmitz P, Bianchin M, Izquierdo I, Medina JH (1997) Involvement of hippocampal cAMP/cAMP-dependent protein kinase signaling pathways in a late memory consolidation phase of aversively motivated learning in rats. Proc Natl Acad Sci U S A 94:7041-7046.

Bilbo SD, Newsum NJ, Sprunger DB, Watkins LR, Rudy JW, Maier SF (2007) Differential effects of neonatal handling on early life infection-induced alterations in cognition in adulthood. Brain Behav Immun 21:332-342.

Blendy JA, Kaestner KH, Schmid W, Gass P, Schutz G (1996) Targeting of the CREB gene leads to up-regulation of a novel CREB mRNA isoform. EMBO J 15:1098-1106.

Bowlby J (1954) The effect of separation from the mother in early life. Ir J Med Sci 6:121-126.

Breier A, Kelsoe JR Jr, Kirwin PD, Beller SA, Wolkowitz OM, Pickar D (1988) Early parental loss and development of adult psychopathology. Arch Gen Psychiatry 45:987-993.

Champagne DL, de Kloet ER, Joels M (2009) Fundamental aspects of the impact of glucocorticoids on the (immature) brain. Semin Fetal Neonatal Med 14:136-142.

Colombo PJ, Brightwell JJ, Countryman RA (2003) Cognitive strategyspecific increases in phosphorylated cAMP response elementbinding protein and c-Fos in the hippocampus and dorsal striatum. J Neurosci 23:3547-3554.

Crain B, Cotman C, Taylor D, Lynch G (1973) A quantitative electron microscopic study of synaptogenesis in the dentate gyrus of the rat. Brain Res 63:195-204.
De Bellis MD (2001) Developmental traumatology: the psychobiological development of maltreated children and its implications for research, treatment, and policy. Dev Psychopathol 13:539-564.

Denenberg VH (1963) Early experience and emotional development. Sci Am 208:138-146.

Dudai Y, Carruthers M (2005) The Janus face of Mnemosyne. Nature 434:567.

Escorihuela RM, Tobena A, Fernandez-Teruel A (1995) Environmental enrichment and postnatal handling prevent spatial learning deficits in aged hypoemotional (Roman high-avoidance) and hyperemotional (Roman low-avoidance) rats. Learn Mem 2:40-48.

Frank DA, Greenberg ME (1994) CREB: a mediator of long-term memory from mollusks to mammals. Cell 79:5-8.

Garoflos E, Panagiotaropoulos T, Pondiki S, Stamatakis A, Philippidis E, Stylianopoulou $F(2005)$ Cellular mechanisms underlying the effects of an early experience on cognitive abilities and affective states. Ann Gen Psychiatry 4:8.

Godfrey KM, Lillycrop KA, Burdge GC, Gluckman PD, Hanson MA (2007) Epigenetic mechanisms and the mismatch concept of the developmental origins of health and disease. Pediatr Res 61 $(5 \mathrm{Pt}$ 2):5R-10R.

Gold AE, Kesner RP (2005) The role of the CA3 subregion of the dorsal hippocampus in spatial pattern completion in the rat. Hippocampus $15: 808-814$

Hall J, Thomas KL, Everitt BJ (2001) Fear memory retrieval induces CREB phosphorylation and Fos expression within the amygdala. Eur J Neurosci 13:1453-1458.

Harlow HF (1965) Total social isolation: effects on macaque monkey behavior. Science 148:666.

Heim C, Shugart M, Craighead WE, Nemeroff CB (2010) Neurobiological and psychiatric consequences of child abuse and neglect. Dev Psychobiol 52:671-690.

Hulshof HJ, Novati A, Sgoifo A, Luiten PG, den Boer JA, Meerlo P (2011) Maternal separation decreases adult hippocampal cell proliferation and impairs cognitive performance but has little effect on stress sensitivity and anxiety in adult Wistar rats. Behav Brain Res 216:552-560.

Impey S, Smith DM, Obrietan K, Donahue R, Wade C, Storm DR (1998) Stimulation of cAMP response element (CRE)-mediated transcription during contextual learning. Nat Neurosci 1:595-601.

Izquierdo LA, Barros DM, Vianna MR, Coitinho A, deDavid e Silva T, Choi H, Moletta B, Medina JH, Izquierdo I (2002) Molecular pharmacological dissection of short- and long-term memory. Cell Mol Neurobiol 22:269-287.

Izquierdo LA, Viola H, Barros DM, Alonso M, Vianna MR, Furman M, Levi de Stein M, Szapiro G, Rodrigues C, Choi H, Medina JH, Izquierdo I (2001) Novelty enhances retrieval: molecular mechanisms involved in rat hippocampus. Eur J Neurosci 13:1464-1467.

Kendler KS (2010) Genetic and environmental pathways to suicidal behavior: reflections of a genetic epidemiologist. Eur Psychiatry 25:300-303.

Kesner RP, Lee I, Gilbert P (2004) A behavioral assessment of hippocampal function based on a subregional analysis. Rev Neurosci 15:333-351.

Kida S, Josselyn SA, Pena de Ortiz S, Kogan JH, Chevere I, Masushige S, Silva AJ (2002) CREB required for the stability of new and reactivated fear memories. Nat Neurosci 5:348-355.

Lamprecht $R$ (1999) CREB: a message to remember. Cell Mol Life Sci 55:554-563.

Lehmann J, Pryce CR, Bettschen D, Feldon J (1999) The maternal separation paradigm and adult emotionality and cognition in male and female Wistar rats. Pharmacol Biochem Behav 64:705-715.

Leon M (1992) The neurobiology of filial learning. Annu Rev Psychol 43:377-398.

Levine S (1967) Maternal and environmental influences on the adrenocortical response to stress in weanling rats. Science 156:258260. 
Levine S (2001) Primary social relationships influence the development of the hypothalamic-pituitary-adrenal axis in the rat. Physiol Behav 73:255-260.

Lippmann M, Bress A, Nemeroff CB, Plotsky PM, Monteggia LM (2007) Long-term behavioural and molecular alterations associated with maternal separation in rats. Eur J Neurosci 25:30913098.

Liu FC, Graybiel AM (1996) Spatiotemporal dynamics of CREB phosphorylation: transient versus sustained phosphorylation in the developing striatum. Neuron 17:1133-1144.

Loy R, Moore RY (1979) Ontogeny of the noradrenergic innervation of the rat hippocampal formation. Anat Embryol (Berl)157(3):243253.

Lyons-Ruth K, Dutra L, Schuder MR, Bianchi I (2006) From infant attachment disorganization to adult dissociation: relational adaptations or traumatic experiences? Psychiatr Clin North Am 29:6386 , viii.

McEwen BS (2001) From molecules to mind. Stress, individual differences, and the social environment. Ann N Y Acad Sci 935:42-49.

McLean JH, Harley CW, Darby-King A, Yuan Q (1999) pCREB in the neonate rat olfactory bulb is selectively and transiently increased by odor preference-conditioned training. Learn Mem 6:608-618.

Meaney MJ, Aitken DH, van Berkel C, Bhatnagar S, Sapolsky RM (1988) Effect of neonatal handling on age-related impairments associated with the hippocampus. Science 239:766-768.

Mischel W, Shoda Y, Rodriguez MI (1989) Delay of gratification in children. Science 244:933-938.

Mizuno M, Yamada K, Maekawa N, Saito K, Seishima M, Nabeshima $\mathrm{T}$ (2002) CREB phosphorylation as a molecular marker of memory processing in the hippocampus for spatial learning. Behav Brain Res 133:135-141.

Moncada D, Viola H (2006) Phosphorylation state of CREB in the rat hippocampus: a molecular switch between spatial novelty and spatial familiarity? Neurobiol Learn Mem 86:9-18.

Montminy M (1997a) Transcriptional activation. Something new to hang your HAT on. Nature 387:654-655.

Montminy M (1997b) Transcriptional regulation by cyclic AMP. Annu Rev Biochem 66:807-822.

Moriceau S, Raineki C, Holman JD, Holman JG, Sullivan RM (2009) Enduring neurobehavioral effects of early life trauma mediated through learning and corticosterone suppression. Front Behav Neurosci 3:22.

Moriceau S, Sullivan RM (2005) Neurobiology of infant attachment. Dev Psychobiol 47:230-242.

Moriceau S, Sullivan RM (2006) Maternal presence serves as a switch between learning fear and attraction in infancy. Nat Neurosci 9:1004-1006.

Moriceau S, Wilson DA, Levine S, Sullivan RM (2006) Dual circuitry for odor-shock conditioning during infancy: corticosterone switches between fear and attraction via amygdala. J Neurosci 26:67376748.

Nair A, Vadodaria KC, Banerjee SB, Benekareddy M, Dias BG, Duman RS, Vaidya VA (2007) Stressor-specific regulation of distinct brainderived neurotrophic factor transcripts and cyclic AMP response element-binding protein expression in the postnatal and adult rat hippocampus. Neuropsychopharmacology 32:1504-1519.

Nelson EE, Herman KN, Barrett CE, Noble PL, Wojteczko K, Chisholm K, Delaney D, Ernst M, Fox NA, Suomi SJ, Winslow JT, Pine DS (2009) Adverse rearing experiences enhance responding to both aversive and rewarding stimuli in juvenile rhesus monkeys. Biol Psychiatry 66:702-704.

Niwa M, Matsumoto Y, Mouri A, Ozaki N, Nabeshima T (2010) Vulnerability in early life to changes in the rearing environment plays a crucial role in the aetiopathology of psychiatric disorders. Int J Neuropsychopharmacol 18:1-19.

O'Keefe J, Nadel L (1978) The hippocampus as a cognitive map. Oxford, UK: Oxford University Press.
Oomen CA, Soeters H, Audureau N, Vermunt L, van Hasselt FN, Manders EM, Joëls M, Lucassen PJ, Krugers H (2010) Severe early life stress hampers spatial learning and neurogenesis, but improves hippocampal synaptic plasticity and emotional learning under high-stress conditions in adulthood. J Neurosci 30:66356645.

Panagiotaropoulos T, Diamantopoulou A, Stamatakis A, Dimitropoulou M, Stylianopoulou F (2009) Learning of a T-maze by rat pups when contact with the mother is either permitted or denied. Neurobiol Learn Mem 91:2-12.

Paxinos G, Watson C (1986) The rat brain in stereotaxic coordinates, $2^{\text {nd }}$ edition. San Diego, CA: Academic Press.

Porte Y, Buhot MC, Mons NE (2008) Spatial memory in the Morris water maze and activation of cyclic AMP response element-binding (CREB) protein within the mouse hippocampus. Learn Mem 15: 885-894.

Pryce CR, Bettschen D, Nanz-Bahr NI, Feldon J (2003) Comparison of the effects of early handling and early deprivation on conditioned stimulus, context, and spatial learning and memory in adult rats. Behav Neurosci 117:883-893.

Raineki C, De Souza MA, Szawka RE, Lutz ML, De Vasconcellos LF, Sanvitto GL, Izquierdo I, Bevilaqua LR, Cammarota M, Lucion AB (2009) Neonatal handling and the maternal odor preference in rat pups: involvement of monoamines and cyclic AMP response element-binding protein pathway in the olfactory bulb. Neuroscience 159:31-38.

Ramachandra R, Subramanian T (2011) Atlas of the neonatal rat brain. Boca Raton, FL: CRC Press.

Richards M, Wadsworth ME (2004) Long term effects of early adversity on cognitive function. Arch Dis Child 89:922-927.

Ruedi-Bettschen D, Zhang W, Russig H, Ferger B, Weston A, Pedersen EM, Feldon J, Pryce CR (2006) Early deprivation leads to altered behavioural, autonomic and endocrine responses to environmental challenge in adult Fischer rats. Eur J Neurosci 24: 2879-2893.

Rutter M, Maughan B (1997) Psychosocial adversities in childhood and adult psychopathology. J Pers Disord 11:4-18.

Schable S, Poeggel G, Braun K, Gruss M (2007) Long-term consequences of early experience on adult avoidance learning in female rats: role of the dopaminergic system. Neurobiol Learn Mem $87: 109-122$.

Schulman H (1995) Protein phosphorylation in neuronal plasticity and gene expression. Curr Opin Neurobiol 5:375-381.

Schulz D, Buddenberg T, Huston JP (2007) Extinction-induced "despair" in the water maze, exploratory behavior and fear: effects of chronic antidepressant treatment. Neurobiol Learn Mem 87:624634.

Schulz D, Topic B, De Souza Silva MA, Huston JP (2004) Extinctioninduced immobility in the water maze and its neurochemical concomitants in aged and adult rats: a possible model for depression? Neurobiol Learn Mem 82:128-141.

Schwandt ML, Lindell SG, Sjoberg RL, Chisholm KL, Higley JD, Suomi SJ, Heilig M, Barr CS (2010) Gene-environment interactions and response to social intrusion in male and female rhesus macaques. Biol Psychiatry 67:323-330.

Shionoya K, Moriceau S, Lunday L, Miner C, Roth TL, Sullivan RM (2006) Development switch in neural circuitry underlying odormalaise learning. Learn Mem 13:801-808.

Stamatakis A, Pondiki S, Kitraki E, Diamantopoulou A, Panagiotaropoulos T, Raftogianni A, Stylianopoulou F (2008) Effect of neonatal handling on adult rat spatial learning and memory following acute stress. Stress 11:148-159.

Stevens CF (1994) CREB and memory consolidation. Neuron 13: $769-770$.

Sullivan RM, Landers M, Yeaman B, Wilson DA (2000) Good memories of bad events in infancy. Nature 407:38-39. 
Suomi SJ, Harlow HF, Domek CJ (1970) Effect of repetitive infantinfant separation of young monkeys. J Abnorm Psychol 76: $161-172$.

Tang AC (2001) Neonatal exposure to novel environment enhances hippocampal-dependent memory function during infancy and adulthood. Learn Mem 8:257-264.

Tang AC, Akers KG, Reeb BC, Romeo RD, McEwen BS (2006) Programming social, cognitive, and neuroendocrine development by early exposure to novelty. Proc Natl Acad Sci U S A 103: 15716-15721.

Taubenfeld SM, Wiig KA, Bear MF, Alberini CM (1999) A molecular correlate of memory and amnesia in the hippocampus. Nat Neurosci 2:309-310.

Vallee M, MacCari S, Dellu F, Simon H, Le Moal M, Mayo W (1999) Long-term effects of prenatal stress and postnatal handling on age-related glucocorticoid secretion and cognitive performance: a longitudinal study in the rat. Eur J Neurosci 11:2906-2916.

Vianna MR, Alonso M, Viola H, Quevedo J, de Paris F, Furman M, de Stein ML, Medina JH, Izquierdo I (2000) Role of hippocampal signaling pathways in long-term memory formation of a nonassociative learning task in the rat. Learn Mem 7:333-340.

Viola H, Furman M, Izquierdo LA, Alonso M, Barros DM, de Souza MM, Izquierdo I, Medina JH (2000) Phosphorylated cAMP re- sponse element-binding protein as a molecular marker of memory processing in rat hippocampus: effect of novelty. J Neurosci 20:RC112, 1-5.

Wang H, Hu Y, Tsien JZ (2006) Molecular and systems mechanisms of memory consolidation and storage. Prog Neurobiol 79:123-135.

Weinfield NS, Sroufe LA, Egeland B (2000) Attachment from infancy to early adulthood in a high-risk sample: continuity, discontinuity, and their correlates. Child Dev 71:695-702.

Winograd M, Viola H (2004) Detection of novelty, but not memory of spatial habituation, is associated with an increase in phosphorylated CAMP response element-binding protein levels in the hippocampus. Hippocampus 14:117-123.

Yin JC, Tully T (1996) CREB and the formation of long-term memory. Curr Opin Neurobiol 6:264-268.

Yin JC, Wallach JS, Del Vecchio M, Wilder EL, Zhou H, Quinn WG, Tully $T$ (1994) Induction of a dominant negative CREB transgene specifically blocks long-term memory in Drosophila. Cell 79:49-58.

Zaharia MD, Kulczycki J, Shanks N, Meaney MJ, Anisman H (1996) The effects of early postnatal stimulation on Morris water-maze acquisition in adult mice: genetic and maternal factors. Psychopharmacol (Berl) 128:227-239. 